\title{
Recovery and Restructuring induced by Fission Energy lons in High Burnup Nuclear Fuel
}

M. Kinoshita ${ }^{1,2,3}$, K. Yasunaga ${ }^{4}$, T. Sonoda ${ }^{2}$, A. Iwase ${ }^{5}$, N. Ishikawa ${ }^{1}$, M. Sataka ${ }^{1}$, K. Yasuda $^{4}$,

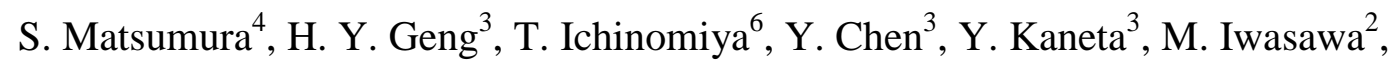
T. Ohnuma ${ }^{2}$, Y. Nishiura ${ }^{6}$, J. Nakamura $^{1}$, Hj Matzke $^{7}$

${ }^{1}$ JAEA Japan Atomic Energy Agency, Tokai-mura, Naka-gun, Ibaraki-ken, 319-1195, Japan; ${ }^{2}$ Central Research Institute of Electric Power Industry, 2-11-1 Iwadokita, Komae-shi Tokyo, 201-8511, Japan; ${ }^{3}$ School of Engineering, The University of Tokyo, Hongo 7-3-1, Bunkyo-ku, Tokyo 113-8656, Japan; ${ }^{4}$ Faculty of Engineering, Kyushu University, 744 Motooka, Nishi-ku, Fukuoka-shi, 819-0395, Japan; ${ }^{5}$ Dept. of Material Science, Osaka Prefecture University, 1-1 Gakuen-cho, Nakaku, Sakai, Osaka 599-8531, Japan; ${ }^{6}$ Research Institute for Electronic Science (RIES), Hokkaido University, Kitaku, Sapporo city, 060-0819, Japan, ${ }^{7}$ Academy of Ceramics, Institute of Trans-Uranium Elements, Karlsruhe, Germany.

\section{Abstract}

In light water commercial reactors, extensive change of grain structure was found at high burnup ceramic fuels. The mechanism is driven by bombardment of fission energy fragments and studies were conducted by combining accelerator based experiments and computer science. Specimen of $\mathrm{CeO}_{2}$ was used as simulation material of fuel ceramics. With swift heavy ion (Xe) irradiation on $\mathrm{CeO}_{2}$, with $210 \mathrm{MeV}$, change of valence charge and lattice deviation of cations were observed by XPS and XRD. Combined irradiations of Xe implantation and swift heavy ion irradiation successfully produced sub-micrometer sized subgrains, similar as that observed in commercial fuels. Studying components of mechanism scenarios, with first principle calculations using the VASP code, we found stable hyperstoichiometric defect structures of $\mathrm{UO}_{2+\mathrm{x}}$. Molecular dynamics studies revealed stability of $\mathrm{Xe}$ planar defects and also found rapid transport mode of oxygen-vacancy clusters. 


\section{Introduction}

The ceramic fuel, $\mathrm{UO}_{2}$, has been used for Light Water Reactors nearly 50 years. In this industry, fuel materials were developed by fully experience-based study, which is known as "Cook and Look" attitude. This strategy had been proper and inevitable since the reactor fuels show typical complex behavior, which includes chemical reactions, phase changes and especially defect production and also recovery cycles which are driven by fission-energy fragments.

Recently the industry is trying to go to the next step of fuel burnup. However, as the burnup exceeds around $70 \mathrm{MWd} / \mathrm{kgU}$, extensive development of porosity was observed at the low temperature region, peripheral of the fuel pellets $[1,2,3,4,5,6]$. As it is not a simple thermal process, empirical approaches such as lowering heat rating and decreasing operating temperature are not effective measures to control restructuring performance of high burnup fuel.

In order to overcome limitation of the traditional development approach, it was started to tie up academic activities and industrial research, providing higher resolution in mechanism study, combining accelerator irradiations and computer-science studies. This paper describes present progress of this activity.

\section{Strategy}

The mission of the program, NXO (New Crossover Project), is to find a mechanism of the restructuring and give methodology to predict and to control the restructuring. Experimentally the first target is to re-produce the principal process of the rim-structure formation outside of the reactor. The process is unique and it must be strictly focused based on the accumulated knowledge of the real fuel behavior. Accelerators are used for two ways; (1) to provide fission induced damaging process, (2) to introduce fission products in the matrix. Here Xe is the most important key fission product due to its large fission yield and special effects in the cation lattice. The fluorite-structured ceramics, $\mathrm{CeO}_{2}$, is selected as the irradiation target to simulate $\mathrm{UO}_{2}$ avoiding inconvenient restrictions. High resolution microscopes of TEM and SEM are the key devices to investigate local restructuring processes. Synchrotron radiation and related methods, and XRD, Raman spectroscopy are utilized to identify statistically dominant processes. 
The computational studies include (1) construction of mathematical framework, (2) simulation model and code development, (3) analysis calculations and development of tools. Firstly we have a mathematical perspective. The emergence of the structure, coming from lattice defects and precipitations, are categorized by special dimensions of 1D, 2D, 3D. Target is to describe the critical moments of breaking symmetry, periodicity, local conservation, and special continuity. The order parameter of Landau theory is a typical example materialized from mathematical invariants. The simulations are devoted to understand the restructuring process. Here, it is not intended to have engineering computations applied for designing artifacts or licensing evaluation. The principal objective is to find competing processes and key interactions in the restructuring. Finding the process scenario is the expected contribution. In the past experience, spending years to study the mechanism, many process scenarios were proposed without verification. Presently the industry has no reliable scenario for prediction. It is because the experimental observations, such as TEM pictures at PIE, give only snapshots of the process. Science based simulations may provide confident time-sequence scenario of the snapshots provided by industrial research. The mission of accelerator based simulation and computation are to provide scientific working field under simulating environment of the complex, phase-changing behavior of fuel material in nuclear reactor.

\section{Experimental study by accelerators}

\section{(1) Electron irradiation}

The specimen, $\mathrm{CeO}_{2}$ pellets, were prepared by compacting $\mathrm{CeO}_{2}$ powders by pressing and subsequent sintering. The applied pressure and sintering temperature were ranged such as 50 $\mathrm{MPa}, 150 \mathrm{MPa}, 1400^{\circ} \mathrm{C}$ (8hours), $1600{ }^{\circ} \mathrm{C}$ (12 hours). In the $\mathrm{CeO}_{2}$ specimen, nucleation and growth process of defect clusters has been investigated in situ under electron irradiation. In-situ TEM observation revealed that energetic electrons with energies from 200 to $1000 \mathrm{keV}$ induce defect clusters in $\mathrm{CeO}_{2}$ lying on $\{111\}$ planes with Burgers vector parallel to the <111> directions (Fig.1). The defect clusters are identified as interstitial-type using the inside-outside contrast method. It is proposed to be oxygen ion interstitial clusters formed by selective displacements of atoms from the oxygen sub-lattice [7]. 


\section{(2) Swift heavy ion irradiation}

The effects of fission track on $\mathrm{CeO}_{2}$ is investigated by swift ion irradiation and cross-sectional observation by TEM (Fig.2). Accelerator irradiation was made with Xe at energy from 70 to $210 \mathrm{MeV}$. The threshold, $16 \mathrm{keV} / \mathrm{nm}$, of the electronic stopping power was identified to have the tracks observable. This threshold was not dependent on irradiation temperature up to 800 ${ }^{\circ} \mathrm{C}$. The observed diameter by TEM ranged from $9 \mathrm{~nm}$ at RT to $7 \mathrm{~nm}$ at $800{ }^{\circ} \mathrm{C}$ at surface of the specimen, depending on irradiation temperature [8].

In order to investigate the effect of overlapping irradiation XRD measurement was performed. Irradiation was made by $200 \mathrm{MeV}$ Au and obtained three observations; (1) The peak angle of XRD shifted to smaller value, which corresponds to dilatation of lattice parameter, (2) broadening the peak, shifting the peak position and subsequent narrowing of it after saturation indicate that the cation sub-lattice itself did not change the original fluorite structure by the passing of tracks, (3) shifting of the peak over the two positions could be explained by discrete change of cation charge from $4+$ to $3+$ in Cerium oxide by the high fluence irradiation. The shifting of valence charge was also confirmed by XPS measurement at synchrotron radiation and the results are presented separately [9].

The ultimate project target of the accelerator irradiation is the reproduction of the grain subdivision process observed at high burnup fuel in the reactor. With $\mathrm{CeO}_{2}$, implantation of $\mathrm{Xe}$ at $285 \mathrm{keV}$ gave high inventory of Xe atoms and tangled dislocations, both placed locally around $100 \mathrm{~nm}$ below the specimen surface. Successively $210 \mathrm{MeV}$ Xe irradiation was performed, giving electronic excitations and some of direct nuclear displacements, which simulates passing of fission fragments and activating the target process in the reactor. Highvoltage transmission electron microscopy revealed formation of sub-grains with diameter of below $200 \mathrm{~nm}$ vicinity at grain-boundaries and porosities [11].

\section{Computational study}

Molecular dynamics simulation was conducted to investigate possible meta-stable states and kinetic processes connecting that states, which are created and driven by local energy deposit of fission energy particles. Selection of the analysis target is heuristic and it is based on observations at the real fuel investigations and the scenarios of possible mechanisms. 
Using pair potential model, we studied the planar clustering of Xe aggregating in $\mathrm{UO}_{2}$ [12] (Fig. 3). Providing a quasi-layer distribution of Xe initially, our simulations showed it would be stable even after the melting of $\mathrm{UO}_{2}$. The Xe interstitials in $\mathrm{UO}_{2}$ are in a liquid state and have a layer modulation of density perpendicular to [111] interface, which (1) has a tendency to connect all Xe atoms together, except the case with low Xe density, no isolated Xe cluster was observed; (2) has almost the same layer distances as the solid part of $\mathrm{UO}_{2}$; (3) maintains the planar shape beyond four layers at least. After releasing these Xe out, a loop of planar defects left in the $\mathrm{UO}_{2}$ matrix. The collapsed structure of this loop may give initiation point of the grain sub-division.

The first principle calculation was applied to investigate ordering of oxygen in sub-lattice of $\mathrm{UO}_{2}$, using the VASP code $[13,14,15]$. In order to obtain required accuracy for investigation of localization of f-electrons in the plane wave based method, after extensive trial of the approximation method, we adapted LSDA+U and succeeded to investigate ordering of excess oxygen atoms [15]. Calculations revealed that $\mathrm{U}_{6} \mathrm{O}_{12}$, cuboctahedron (COT), has low formation energy and it may have important role at the final quenching stage after passing of fission tracks (Fig. 4). This result gives more precise understanding how the anisotropic structure of $\mathrm{U}_{4} \mathrm{O}_{9}$ is developed and it is expected that some of observed planar faults of irradiated fuel may be caused by activated process including COT formation.

The dynamics of anion (Oxygen) point defects in $\mathrm{UO}_{2}$ was investigated by simulations using the method of temperature accelerated dynamics (TAD) developed in Loss Alamos Laboratory [16]. Usually it is known that oxygen vacancies are moving much faster than that of the interstitial. However, it is only in the case of single defects. Under irradiation we have high energy defect complex at certain generation rate. Using the TAD code, we found that Oxygen clusters made from two interstitials diffuse as fast as vacancies [17]. In our case, using the Basak potential, the stable structure is one vacancy surrounded by three interstitials. This cluster migrates through a saddle structure which includes two interstitials. It is well known that clustered defects in metals show much faster migration than the single point defects [18]. The result of TAD calculation shows that such phenomena can occur also in ceramic materials. The observed behavior of oxygen in fluorite structure of $\mathrm{UO}_{2}$ indicates that oxygen sub-lattice activates repairing processes of cation sub-lattice which is responsible for intactness of the $\mathrm{UO}_{2}$ crystal structure. 


\section{Summary}

In order to have scientific control and prediction capability of the use of nuclear fuel, motivated by industrial need to study high burnup rim-structure formation, the new cross over project (NXO) of a cooperation of academy and industry, had been initiated. The target is to get over "Cook and Look" attitude, the works totally based on experience, because it is not enough in the scope of modern science and engineering.

Firstly, accelerator based simulation is leading the whole project, challenging to reproduce the target phenomenon, grain sub-division, observed in the reactors. The irradiation by accelerator is expected to give sequence information of the processes which stick together snapshot-observations obtained in the industrial research. Subsequent irradiation of implantation of $\mathrm{Xe}$ and swift ion (also $\mathrm{Xe}$ ) irradiation on $\mathrm{CeO}_{2}$ reproduced sub-grain formation being very similar as the restructuring in high burnup fuels. Pre-installed dislocation network was recovered by overlapping of swift ion tracks, possibly released to neighbored free surfaces. This is the first achievement of the structure reproduction which is under control of the microscopic parameters, both of dislocation density and Xe inventory, showing $100 \mathrm{~nm}$ sized grains similar as that observed in commercial fuels.

In the studies of mechanisms, electron irradiation with TEM observation and also measurements by synchrotron radiation and XRD are conducted. Results showed important roles of oxygen behavior at nanometer scale restructuring and recovery. Theoretical and computer-science based studies were applied to investigate effects of repeated overlapping of swift ion tracks. To achieve statistical description of the processes, nano-scale to sub-micron scale computing environment is in progress of development. Molecular dynamics calculations showed existence of meta-stable planar structure of $\mathrm{Xe}$ on [111] plane of $\mathrm{UO}_{2}$. Accelerated molecular dynamics simulation (TAD) revealed significant kinetic performance of metastable oxygen cluster. The first principle calculations for excess oxygen state of $\mathrm{UO}_{2+\mathrm{x}}$ provided several meta-stable configurations. Especially embedded cuboctahedral cluster $\left(\mathrm{U}_{6} \mathrm{O}_{12}\right)$ in $\mathrm{UO}_{2}$ fluorite structure is minimizing energy significantly.

These active behaviors of local clustering bring up emergence of trans-scale structures starting from atom scale through $\mathrm{nm}$ scale to sub-micron scales. Combination of accelerator irradiation and super-computing opens new world in physics and practical engineering to predicts breaking of symmetries periodicities and orders by the emergence of clustering 
structures. Understanding of self-repairing process of materials under irradiation is one of the significant out-comes of this new technology presently becoming available.

\section{Acknowledgement}

This study was financially supported by the Budget for Nuclear Research of the Ministry of Education, Culture, Sports, Science and Technology, based on the screening and counseling by the Atomic Energy Commission.

\section{References}

[1] M. Kinoshita, T. Sonoda, S. Kitajima, A. Sasahara, T. Kameyama, T. Matsumura, E. Kolstad, V.V. Rondinella, C. Ronchi, J.-P. Hiernaut, T. Wiss, F. Kinnart, J. Ejton, D. Papaioannou, Hj. Matzke: Proceedings of the 2004 International Meeting on LWR Fuel Performance, Orlando, Florida, September 19-22 (2004) paper1102.

[2] M.L. Bleiberg, R.M. Berman and B. Lustman, in: Proc. Symp. on Radiation Damage in solids and Reactor Materials, IAEA, Vienna (1963), p. 319.

[4] J.O.Barner, M.E.Cuningham, M.D.Freshley, D.D Lanning, "High Burn-up Effect Program - Final Report", HBEP-61, 1990, Battelle Pacific Northwest Laboratories

[5] D.Baron, B.Bordin-Lhermitte, J-P Piron, IAEA Technical Commitee Meeting on Advances in Pellet Technology for improved Performance and High Burn-up Toranomon Pastral, TOKYO, JAPAN, October 26th - November 1st, 1996

[6] J. Noirot, L. Desgranges, J. Lamontagne, J of Nucl. Mater. 372 (2007) 318-339.

[7] K. Yasunaga, K. Yasuda, S. Matsumura and T. Sonoda, Nucl. Instr. and Meth. B Vol. 250, (2006) 114.

[8] T. Sonoda, M. Kinoshita, Y. Chimi, N. Ishikawa, M. Sataka, and A. Iwase, Nucl. Inst. and Meth. B 250 (2006) 254.

[9] N. Ishikawa a, Y. Chimi, O. Michikami, Y. Ohta, K. Ohhara, M. Lang, R. Neumann,, NI M Physics Research B 266 (2008) 3033. 
[10] A. Iwase, H. Ohno, D. Matsumura, Y. Nishihata, J. Mizuki, N. Ishikawa, Y. Baba, N. Hirao, T. Sonoda, M. Kinoshita, "Study on the microstructure of swift heavy ion irradiated $\mathrm{CrO}_{2}$ by means of synchrotron radiation $\mathrm{X}$-ray photoelectron spectroscopy and Ce K-edge EXAFS spectroscopy” presented at O21, SHIM2008, Lyon, June 2-5, 2008.

[11] K. Yasunaga, T. Sonoda, T. Yamada, K. Yasuda, et al to be published.

[12] H. Y. Geng, Y. Chen, Y. Kaneta and M. Kinoshita, J. Alloys and Comp., 457 (2008)465.

[13] G. Kresse and J. Furthmüller, Phys. Rev. B 54, (1996) 11169.

[14] M. Iwasawa, Y. Chen, Y. Kaneta and T. Ohnuma, Mater. Trans. 47(2006) 2651.

[15] H. Y. Geng, Y. Chen, Y. Kaneta and M. Kinoshita, Phys. Rev. B 75(2007) 54111.

[16] M. R. Sørensen and A. F. Voter, J. Chem. Phys. 112(2000) 9599.

[17] T. Ichinomiya, to be published in J. Nucl. Mater.

[18] S. Ishino, N, Sekimura, and H. Abe, Journal of ASTM International, 4(2007) paper ID: JAI100644.

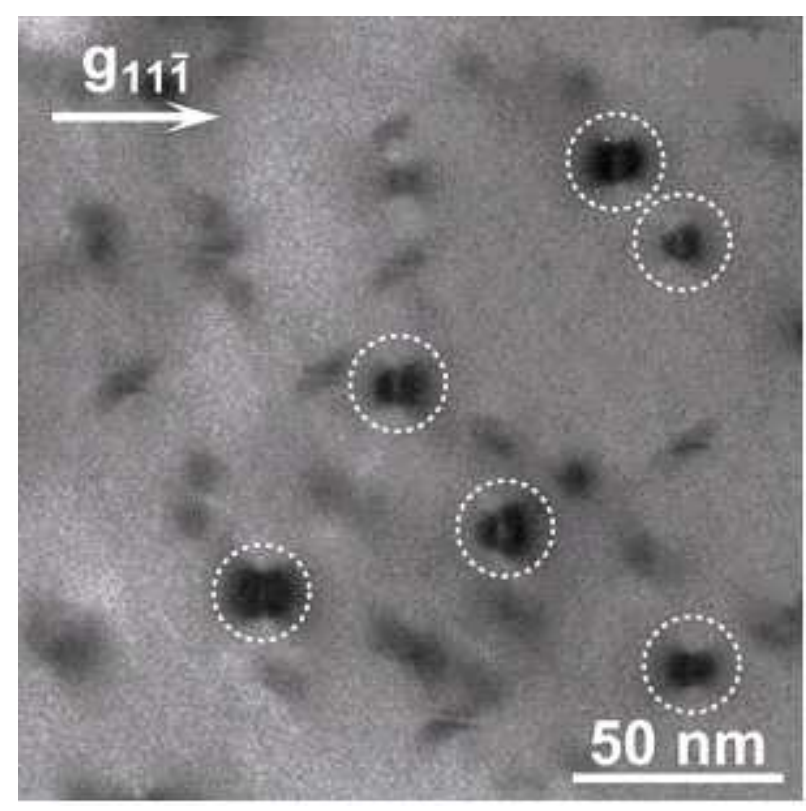

Fig,. Bright field images of the defect clusters in $\mathrm{CeO} 2$ irradiated with $1000 \mathrm{keV}$ electrons at $296 \mathrm{~K}$. The electron beam direction is nearly along the [011] direction ${ }^{7)}$. 


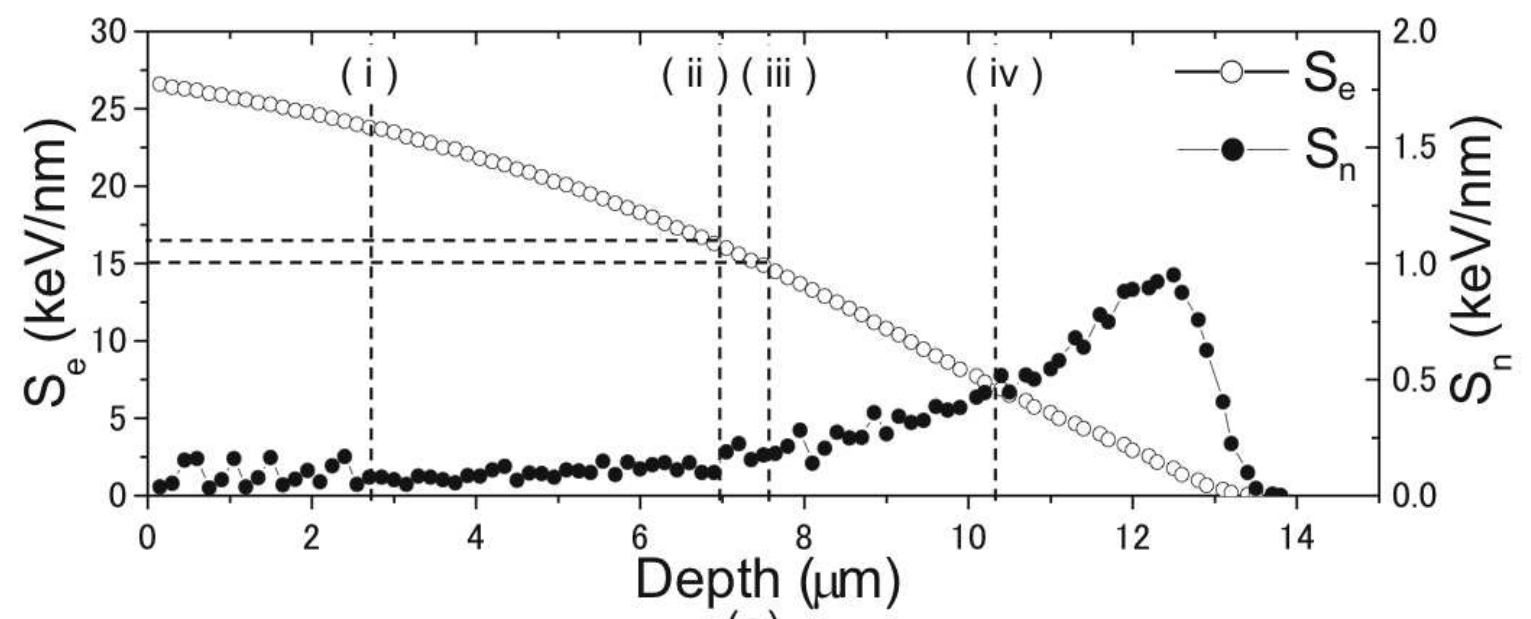

(a)
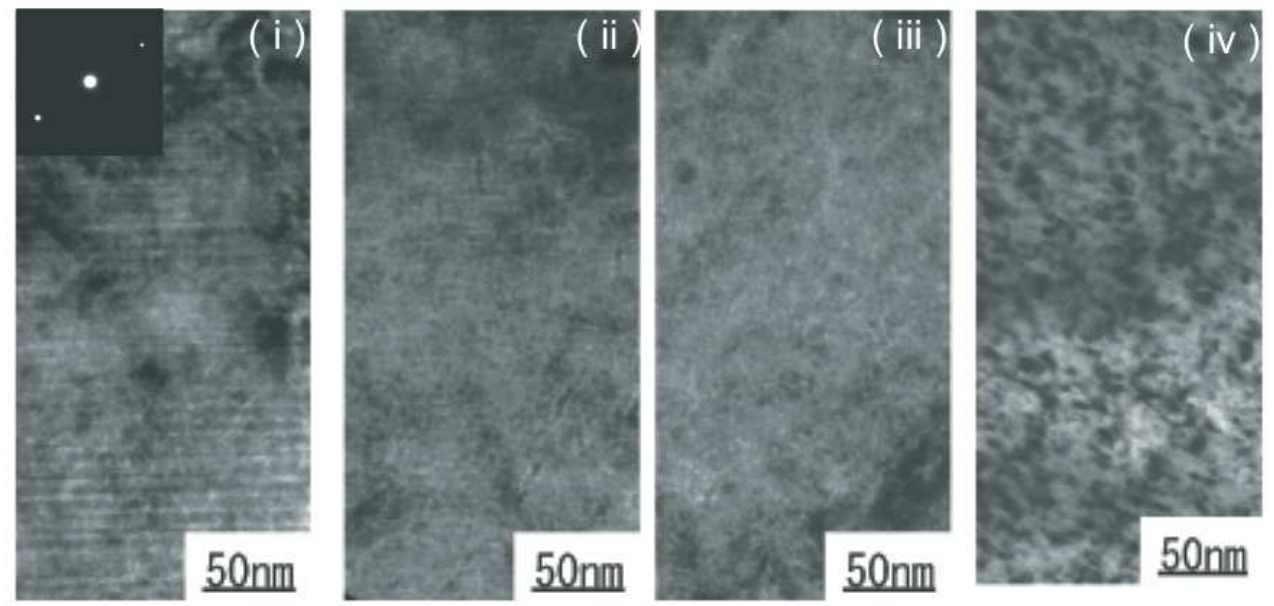

(b)

Fig. 2 Depth profile of electronic stopping power (Se) and nuclear stopping power (Sn) of $210 \mathrm{MeV} \mathrm{Xe}+14$ ion in $\mathrm{CeO} 2$ (a) and the typical TEM images in selected depth positions (b) respectively ${ }^{10)}$. 


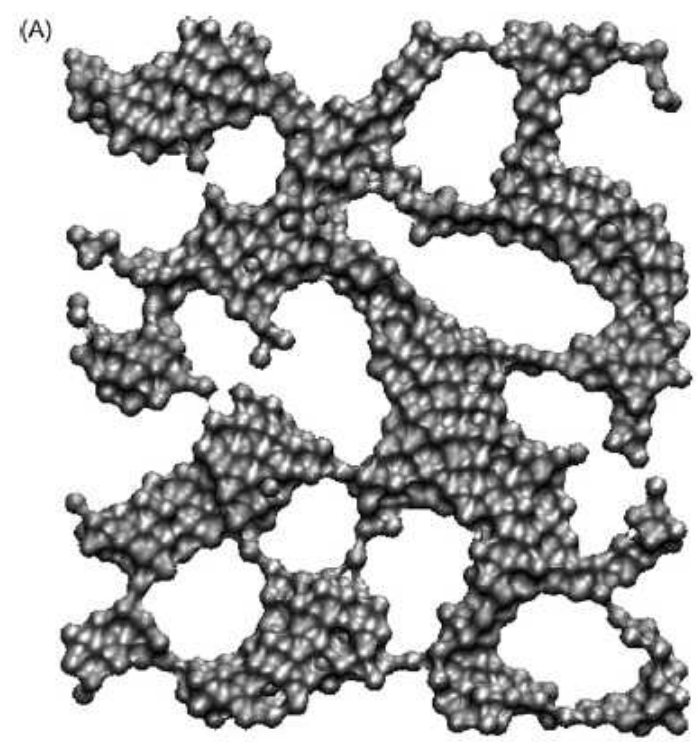

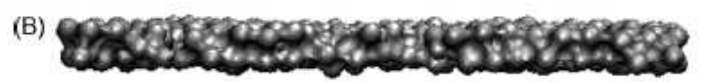

Fig. 3. The structure of two consecutive Xe layers in UO2 simulated at 1000K with an equal amount of xenon to uranium atoms on the same [111] layer. (A) top view and (B) side view ${ }^{11)}$.

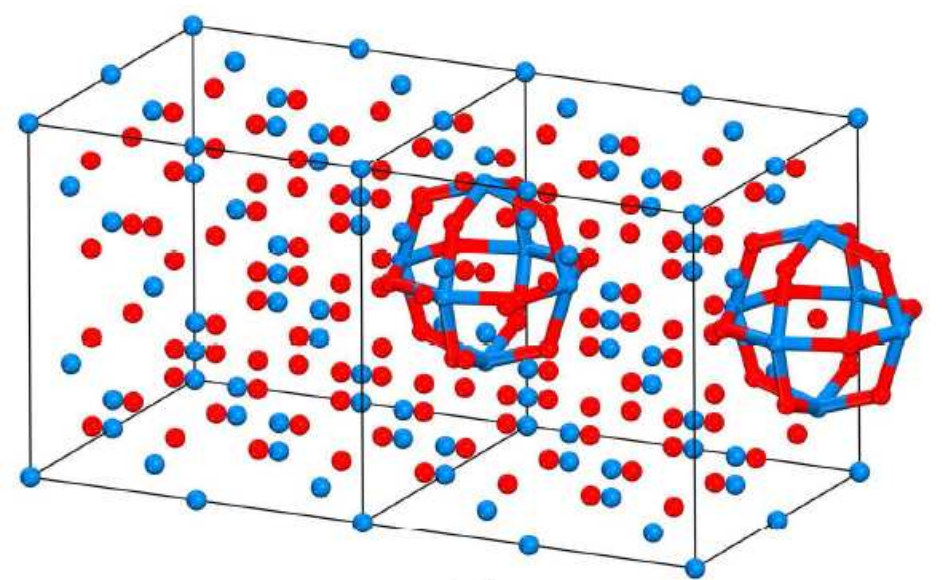

(a)

FIG. 4 Cuboctahedral clusters incorporated in fluorite $\mathrm{UO}_{2}{ }^{14)}$ 